\title{
Structural Basis of Large-Scale Functional Connectivity in the Mouse
}

\author{
Joanes Grandjean, ${ }^{1,2 \star}$ - Valerio Zerbi, ${ }^{3 \star}$ Joshua Henk Balsters, ${ }^{3,4}$ - Nicole Wenderoth, ${ }^{3,5} \uparrow$ and Markus Rudin ${ }^{1,5,6} \dagger$ \\ ${ }^{1}$ Institute for Biomedical Engineering, University and ETH Zürich, 8093 Zürich, Switzerland, ${ }^{2}$ Singapore BioImaging Consortium, Agency for Science \\ Technology and Research, 138667 Singapore, Singapore, ${ }^{3}$ Neural Control of Movement Laboratory, Department of Health Sciences and Technology, ETH \\ Zürich, 8057 Zürich, Switzerland, ${ }^{4}$ Department of Psychology, Royal Holloway University of London, TW20 0EX Egham, Surrey, United Kingdom, \\ ${ }^{5}$ Neuroscience Center Zürich, University and ETH Zürich, 8057 Zürich, Switzerland, and ${ }^{6}$ Institute of Pharmacology and Toxicology, University of Zürich, \\ 8057 Zürich, Switzerland
}

Translational neuroimaging requires approaches and techniques that can bridge between multiple different species and disease states. One candidate method that offers insights into the brain's functional connectivity (FC) is resting-state fMRI (rs-fMRI). In both humans and nonhuman primates, patterns of FC (often referred to as the functional connectome) have been related to the underlying structural connectivity (SC; also called the structural connectome). Given the recent rise in preclinical neuroimaging of mouse models, it is an important question whether the mouse functional connectome conforms to the underlying SC. Here, we compared FC derived from rs-fMRI in female mice with the underlying monosynaptic structural connectome as provided by the Allen Brain Connectivity Atlas. We show that FC between interhemispheric homotopic cortical and hippocampal areas, as well as in cortico-striatal pathways, emerges primarily via monosynaptic structural connections. In particular, we demonstrate that the striatum (STR) can be segregated according to differential rs-fMRI connectivity patterns that mirror monosynaptic connectivity with isocortex. In contrast, for certain subcortical networks, FC emerges along polysynaptic pathways as shown for left and right STR, which do not share direct anatomical connections, but high FC is putatively driven by a top-down cortical control. Finally, we show that FC involving cortico-thalamic pathways is limited, possibly confounded by the effect of anesthesia, small regional size, and tracer injection volume. These findings provide a critical foundation for using rs-fMRI connectivity as a translational tool to study complex brain circuitry interactions and their pathology due to neurological or psychiatric diseases across species.

Key words: functional connectome; mouse; resting-state fMRI; structural connectivity; viral tracing

Significance Statement

A comprehensive understanding of how the anatomical architecture of the brain, often referred to as the "connectome," corresponds to its function is arguably one of the biggest challenges for understanding the brain and its pathologies. Here, we use the mouse as a model for comparing functional connectivity (FC) derived from resting-state fMRI with gold standard structural connectivity measures based on tracer injections. In particular, we demonstrate high correspondence between FC measurements of cortico-cortical and cortico-striatal regions and their anatomical underpinnings. This work provides a critical foundation for studying the pathology of these circuits across mouse models and human patients.

\section{Introduction}

The brain relies on finely tuned and adaptable neuronal networks for the control of perception, cognition, and behavior. The orga-

\footnotetext{
Received Feb. 16, 2017; revised June 20, 2017; accepted June 28, 2017.

Author contributions: J.G., V.Z., N.W., and M.R. designed research; J.G. and V.Z. performed research; J.G., V.Z., and J.B. analyzed data; J.G., V.Z., J.B., N.W., and M.R. wrote the paper.

This work was supported by the Swiss National Science Foundation (Grants 310030_141202 and 310030_160310 to M.R.) and the Swiss Foundation for Excellence and Talent in Biomedical Research (M.R.). V.Z. is supported by ETH Career Seed Grant SEED-42 16-1.

The authors declare no competing financial interests.

*J.G. and V.Z. contributed equally to this work and share first authorship.
}

nization of these networks at the microscale, mesoscale, and macroscale levels are considered essential for the segregation and integration of information throughout the brain (Bullmore and Sporns, 2009; Park and Friston, 2013). Neuronal networks can be identified either at the structural level, that is, represented by the physical presence of neuron cell bodies, dendrites, projecting ax-

†N.W. and M.R. contributed equally to this work and share last authorship.

Correspondence should be addressed to Prof. Dr. Markus Rudin, Institute for Biomedical Engineering, University and ETH Zürich, AIC-ETH HCI D426, Vladimir-Prelog-Weg 4, CH- 8093 Zürich. E-mail: rudin@biomed.ee.ethz.ch. DOI:10.1523/JNEUROSCI.0438-17.2017

Copyright $\odot 2017$ the authors $\quad 0270-6474 / 17 / 378092-10 \$ 15.00 / 0$ 
ons, and synapses connecting two neuronal entities, or at the functional level, that is, by dependencies of spiking and synaptic activities across neurons (Park and Friston, 2013). Such structural and functional measurements of the brain can then be conceptualized by describing ROIs as nodes and interactions between nodes as edges, a framework that has become important for defining the intrinsic architecture of the brain, also referred to as the "connectome," as well as its alterations due to disorders (Bullmore and Sporns, 2009). Information on the functional connectome can be resolved using resting-state fMRI (rs-fMRI). rs-fMRI estimates the statistical interdependence of two brain areas from temporal correlations of fluctuating blood oxygen levels (Biswal et al., 1995; Damoiseaux et al., 2006; De Luca et al., 2006; Biswal et al., 2010). It has been proposed that high functional connectivity (FC) between remote brain areas results from information exchange via anatomical connections, suggesting that emerging functional networks reflects indirectly the architecture of the structural connectome (Honey et al., 2009; van den Heuvel et al., 2009; Zhang et al., 2010). Several approaches are available to assess anatomical connectivity, such as in vivo diffusion-weighted MRI (Basser et al., 1994) and tractography, as well as ex vivo histological cyto-architectonic mapping (Kasthuri and Lichtman, 2007; Lichtman et al., 2008). For human studies, diffusion-weighted MRI-based connectome reconstruction has become the method of choice, being noninvasive and available in many research centers. However, tractography methods have difficulty resolving crossing fibers (Jbabdi et al., 2015) and thus lose their sensitivity in gray matter and do not provide reliable information on track terminations. Anterograde/retrograde neuronal viral tracer studies in animal models are not hindered by crossing fibers and are currently considered the gold standard for determining monosynaptic axonal pathways (Swanson, 1982; Oh et al., 2014; Zingg et al., 2014; Hintiryan et al., 2016). Recently, viral tracers have been used extensively to map the mouse brain's monosynaptic structural connectome at the mesoscale level (Oh et al., 2014; Zingg et al., 2014; Hintiryan et al., 2016). This offers new opportunities to validate noninvasive imaging methods against the anatomical ground truth using a mammalian model, which shares several key principles of network architecture with the human brain (Stafford et al., 2014; van den Heuvel et al., 2016). However, in the past, detailed structurefunction comparisons in the mouse brain have been limited by the achievable rs-fMRI quality. Here, we used advanced MRI technology and protocols (Grandjean et al., 2014; Zerbi et al., 2015) for deriving the brain-wide functional connectome of mouse from high-resolution rs-fMRI acquisitions, which we then compared through a systematic approach with the monosynaptic structural connectome derived from viral tracings (Allen Brain Institute, http://connectivity.brain-map.org/).

Our results confirm that high FC emerges predominantly between monosynaptically connected regions. In addition to strong FC between homotopic areas of left and right isocortex, we show now for the mouse brain that the striatum (STR) can be segregated according to differential rs-fMRI connectivity patterns that mirror monosynaptic structural connectivity (SC) with isocortex. Interestingly, we also found that high FC between subcortical structures of the left and right hemisphere emerges via polysynaptic pathways, suggesting that isocortex might be an important relay area for mediating FC that extends beyond the monosynaptic structural connectome. In contrast, FC along cortico-thalamic pathways was limited, possibly confounded by the effect of anesthesia, ROI size, and tracer injection volume. These results provide a critical foundation for future work that aims to test connectivity changes in mouse models of human diseases to reveal how brain connectivity is altered in pathological phenotypes.

\section{Materials and Methods}

Study design. To investigate the structural substrate underlying FC in the murine brain, rs-fMRI date sets from 14 female C57BL/6 mice were collected and the resulting FC data compared with SC data derived from viral tracer maps from the Allen Brain Institute. The mouse brain functional connectome was obtained using a subset of seeds (238) and target regions (254) taken from the Allen reference atlas using Pearson's correlation (Fisher's $z$ transformed). The agreement between SC and FC was evaluated by the following: (1) partial Spearman's correlations that include region volume as a covariate to account for dominating effects of large seed regions; (2) on the basis of receiver operating characteristic (ROC) curves; and (3) with a "winner-takes-all" analysis labeling each voxel as belonging to the network with the largest connectivity value. Finally, monosynaptic and polysynaptic dependency connections were derived from a graph theory approach comparing the distances (number of edges between two nodes) in thresholded SC and FC maps.

The SC matrix based on viral tracer injections was adapted from $\mathrm{Oh}$ et al. (2014). Briefly, adeno-associated viral anterograde tracers containing genes encoding for enhanced green fluorescent protein were sterotactically injected at different sites in mice. After the injection, 2 weeks were allowed for the protein expression before the animals were killed, the brain extracted, sectioned, imaged with two-photon microscope, reconstructed into 3D fluorescence maps, and transformed into a common reference space. The connectivity was determined from the injection site to the projections by quantifying the fluorescence locally for each ROI and normalizing it with the volume of injection (for further details, see Oh et al., 2014).

Viral-tracer maps resampled at $100 \mu \mathrm{m}^{3}$ were obtained using the query form from the Allen Institute database. Individual experiments were selected as follows: carried in wild-type C57BL/ 6 and with injection volume ranging from 0.0001 to $0.5 \mu$ l. The anatomical reference template was coregistered into the Australian Mouse Brain Mapping Consortium MRI template resampled at $100 \mu \mathrm{m}^{3}$ (http://www.imaging.org.au/AMBMC), using Advanced Normalization Tools (ANTS) with greedy SyN transformation (version 2.1, http://picsl.upenn.edu/software/ants/). The viraltracer maps and the anatomical atlas were then converted into MRI space using the transformations estimated earlier and radiological convention, which represents inverted left and right orientations.

We limited the scope of our analysis to the ontological ROI groups of the Allen atlas that were fully covered by the fMRI volume acquisition: the isocortex, cortical subplate (CTXsp), hippocampal formation (HPF), STR, palladium (PAL) and thalamus (TH).

$f M R I$ data acquisition and preprocessing. Mouse multiecho fMRI data are available online (central.xnat.org, project_ID: ME_epi_mouse). The rs-fMRI functional connectome maps are freely available for consultation (http://doi.org/10.5905/ethz-1007-59).

C57BL/ 6 female mice $(n=14)$ were used in this study. The experiment was performed following the Swiss federal guidelines for animal experimentation and under a license from the Zürich cantonal veterinary office. Animals were caged in standard housing with food and water ad libitum and kept in a $12 \mathrm{~h}$ light/dark cycle. In preparation for the measurements, anesthesia was induced with isoflurane $3.5 \%$ for $4 \mathrm{~min}$ in a $1: 4$ oxygen to air mixture. The animals were endotracheally intubated and positioned on an MR-compatible support. The animals were fixed with ear bars and mechanically ventilated using a ventilator (CWE) with $2 \%$ isoflurane. A cannula was placed in the tail vein for a medetomidine 0.05 $\mathrm{mg} / \mathrm{kg}$ bolus followed $5 \mathrm{~min}$ later by $0.1 \mathrm{mg} / \mathrm{kg} / \mathrm{h}$ infusion and a pancuronium $0.2 \mathrm{mg} / \mathrm{kg}$ bolus followed by $0.4 \mathrm{mg} / \mathrm{kg} / \mathrm{h}$ infusion. After the medetomidine bolus injection, isoflurane was reduced to $1.5 \%$ and further reduced to $0.5 \%$ during infusion. Animal preparation and measurement took $45 \mathrm{~min}$ and all animals recovered fully after the measurements.

The dataset was acquired on a 9.4 $\mathrm{T}$ Bruker scanner equipped with a BGA-S gradient system, a volume coil for excitation, and a $2 \times 2$ phasedarray receiver-only cryogenic coil. Multiecho gradient-echo echo-planar imaging (ME-EPI) were acquired with the following parameters: repetition time $1500 \mathrm{~ms}$, echo time $[11,17,23] \mathrm{ms}$, flip angle $60^{\circ}$, matrix size 


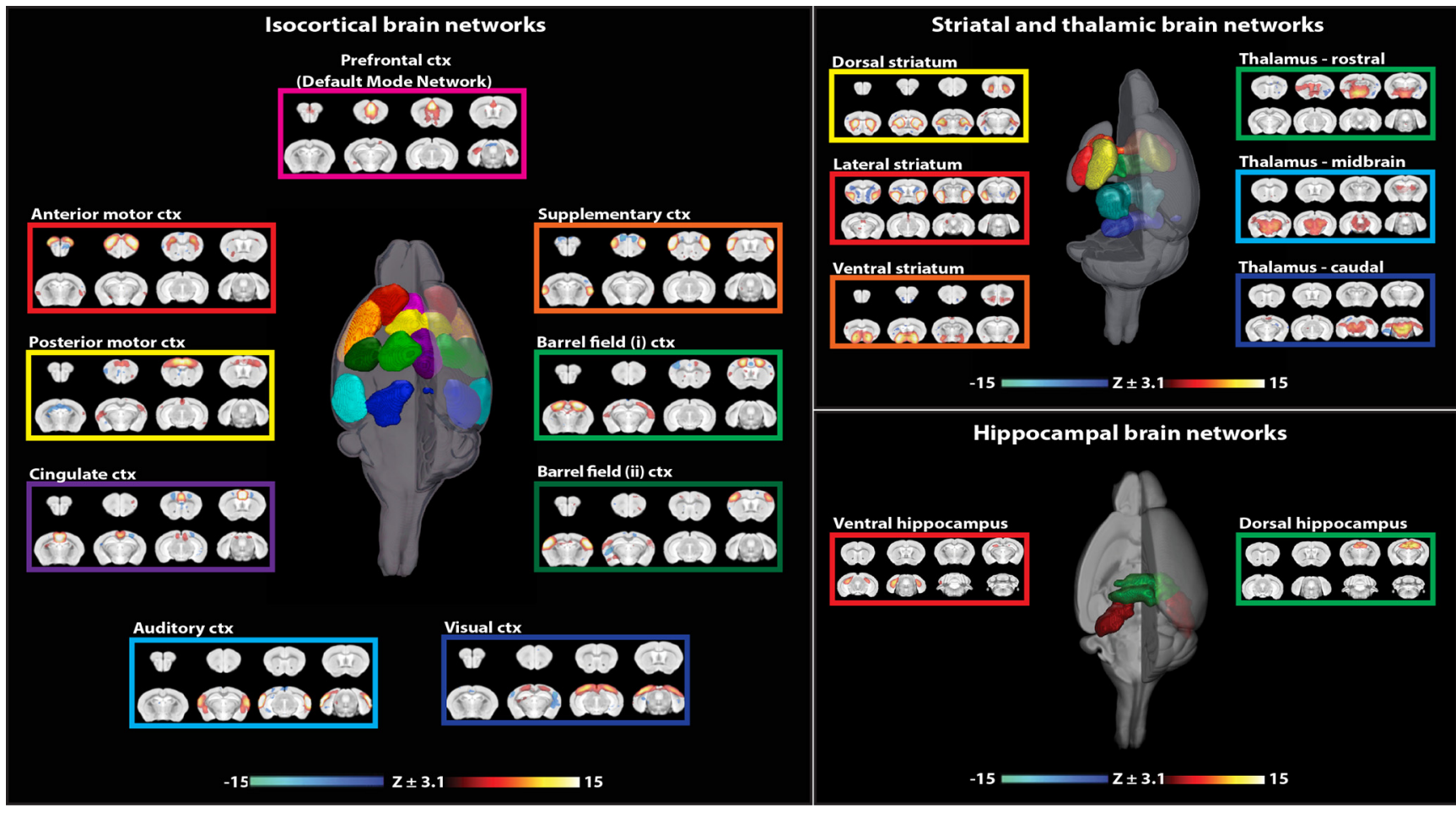

Figure 1. ICA revealed the presence of robust resting-state networks in the mouse brain. Optimized MR acquisition, anesthesia and handling, and image-processing protocols yielded readily defined isocortical, striatal, thalamic, and hippocampal rs-fMRI networks.

$60 \times 30$, field of view $18.2 \times 9 \mathrm{~mm}^{2}$, number of slices 20 , slice thickness $0.3 \mathrm{~mm}$, slice gap $0.05 \mathrm{~mm}, 600$ volumes, acceleration factor 1.4 , horizontal field of view saturation slice to mask the lower portion of the mouse head, 250,000 $\mathrm{Hz}$ bandwidth. Images presented minimal distortions, even at higher echo time.

ME-EPI images were converted to NIFTI format and processed with meica.py script (AFNI_2011_12_21_1014, http://afni.nimh.nih.gov) (Kundu et al., 2012). Briefly, the script performs motion correction, despiking, skull stripping, and an independent component analysis (ICA) decomposition of the 3 echo-separated 4D images. Echo-time dependency was measured in each component and used to discriminate BOLD-related from non-BOLD related components. Components that did not present echo-time signal dependency, that is, that were nonBOLD, were labeled as noise and then regressed to obtain a final denoised $4 \mathrm{D}$ image. The denoised fMRI images were then coregistered to the MRI template using linear affine and nonlinear greedy SyN transformation. We identified large-scale FC networks using an ICA (group-ICA MELODIC, FMRIB Software Library version 5.0, http://fsl.fmrib.ox.ac. $\mathrm{uk}$ ) as a tool to evaluate the quality of the cleaned data. Of the 30 predetermined components, 17 were known cortical and subcortical networks with plausible anatomical locations (Fig. 1) based on definitions outlined previously (Zerbi et al., 2015). Other components displayed spatial maps with irregular clusters not related to anatomical gray matter structures. None of the components related to motion or to vascular structures have been found. This is consistent with previous observations using similar acquisition protocols, but different artifact cleaning methods (Zerbi et al., 2015). Seed-based maps were computed in FSL with the Glm function using the viral injection site and injection volume to define the 238 rsfMRI seeds. FC between each seed and the 254 target regions taken from the Allen reference atlas with the same ontology as used in Oh et al. (2014) was calculated using Pearson's correlation and Fisher's $z$ transformed. Individual $z$-statistic seed-to-target matrices were premasked using a $t$ test to consider only connections significantly different from 0 $[p=0.05$, false discovery rate (FDR) corrected $]$ and group averaged. The correlation between the SC and FC in each individual tracer experiment (i.e., each row in the connectome matrices) was defined by partial Spearman's correlations that include region volume as a covariate to compensate for the common dependency between both tracer-based connectivity and rs-fMRI with regional target volumes (Sethi et al., 2017). The results yielded values of no/weak $(0-0.2)$, weak-to-moderate $(0.2-0.4)$, and moderate-to-strong (0.4-0.6) effects (Cohen, 1988). Nonparametric Spearman's correlation was used because we could not readily assume a linear relationship between tracer projection intensity and FC. In complement to the previous analysis with partial Spearman's correlation analysis using region volume as covariate, ROC curves were computed as described previously (Calabrese et al., 2015) to assess the gross agreement between anatomical macroscale FC and SC. Briefly, SC and FC submatrices were selected from their anatomical parent structures, log transformed, normalized between 0 and 1 , and binarized using a series of 1000 thresholds to keep the $0-100 \%$ of connections. The resulting binary connectivity matrices were compared using ROC analysis with tracerbased connectivity as ground truth. The true positive rate and false positive rate vectors were plotted against each other and the resulting area under the curve (AUC) was used as measure of connectivity similarity between the two metrics. The AUC results were then compared against a null distribution of the same datasets using permutation testing. Permutation testing (1000 iterations) consisted of shuffling the labels of the FC submatrices. In each permutation, the total volume of brain covered by the labels was therefore identical between SC and FC, which excludes volume-based biases in the resulting values.

For every voxel within each mask (right hemisphere isocortex, left hemisphere STR, and left TH), we extracted the transformed connectivity values for each injection site. This gave each voxel a connectivity profile indicating how strong or weak connectivity was at that voxel for each injection site for both rs-fMRI and tracer connectivity. To determine whether resting-state connectivity patterns matched those seen with tracer injections, we performed a winner-takes-all analysis, labeling each voxel as belonging to the network with the largest connectivity value for a set of 20 injection sites distributed on the right isocortical hemisphere as per Oh et al. (2014). To determine whether voxels showed the same connectivity fingerprint using rs-fMRI and tracers (i.e., if voxels showed connectivity profile for injection sites in both modalities), we used Spearman's $\rho$ to correlate connectivity profiles at each voxel. FDR $(p<0.05)$ was used to correct for multiple comparisons. 


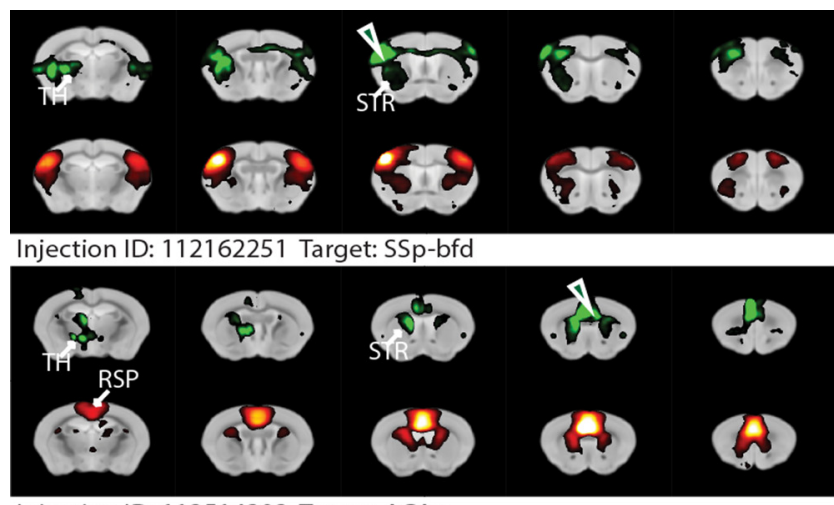

Injection ID: 112514202 Target: ACAv

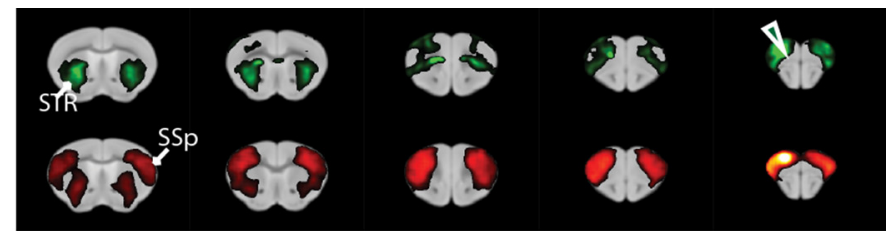

Injection ID: 100140756 Target: MOs

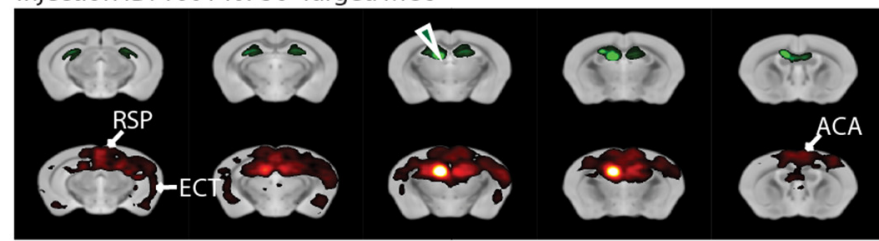

Injection ID: 100141214 Target: DG

2.7

Tracer's intensity projection [A.U.]

rsfMRI connectivity [Z-score]

Figure 2. Qualitative comparison between tracer distribution indicating SC (green; top) and FC pattern derived from rs-fMRI (red; bottom) for four selected injection sites/seeds. The results illustrate a high degree of similarity between the measurements, particularly in ipsilateral cortico-striatal connectivity. A high degree of overlap was also found in contralateral cortico-cortical and hippocampalo-hippocampal connections, whereas cortico-thalamic anatomical projections were not detected by rs-fMRI.

Monosynaptic and polysynaptic dependency connections were derived from a graph theory approach. To this end, we used the symmetrical SC matrix $(81 \times 81$; for ROI list, see Fig. $3-2$, available at 10.1523/JNEUROSCI.0438-17.2017.f3-2) adapted from Oh et al. (2014). The symmetrical FC matrix was obtained by estimating the Pearson's correlation coefficient between the time series extracted from rs-fMRI scans using the same ROI set as for SC. Individual FC matrices were groups averaged without applying any premasking to maintain its original distribution. Both symmetrical matrices were normalized to range $0-100$. Connectivity matrices were binarized using varying threshold $0-100$ by one increment; at each threshold level, the minimal distance separating every ROI pair was estimated using igraph in R (The R Foundation for Statistical Computing, Vienna, Austria). For every threshold level, the interaction between each ROI pair were classified into three categories based on both SC and FC distance matrix: (1) monosynaptic $\mathrm{FC}\left(\right.$ distance $_{\mathrm{SC}}=1$ edge and distance FC $=1$ edge); (2) polysynaptic FC (distance $_{\mathrm{SC}}>1$ edge and distance ${ }_{\mathrm{FC}}=1$ edge); and (3) mismatch (distance $_{\mathrm{SC}}=1$ edge and distance $\mathrm{FC}_{\mathrm{F}}>1$ edge). The connectivity likelihood for each category was established as the incidence for each threshold level.

Statistical analysis. All the correlations, such as between functional and SC data or between the partial Spearman's $\rho$ and the viral injection volume, were tested using a two-way $t$ test testing the hypothesis of no correlation against the alternative that there is a nonzero correlation. For ROC analysis, permutation testing was performed for the ROC-AUC calculation to test their significance against a null distribution, as described in the previous section.

\section{Results}

Overlapping structural and FC in the mouse brain

SC and FC represent different metrics that depict large-scale neuronal architecture. Viral-tracer distributions obtained from the Allen Brain Institute database and rs-fMRI seed maps corresponding to the injection sites were normalized into common spatial coordinates. Voxelwise representation of the viral-tracer distribution and seed-based FC for four selected injection sites/ seeds provide a qualitative representation of the similarities and differences between SC and FC (Fig. 2). For instance, tracer injected into the primary somatosensory area of the barrel field highlighted projections to both ipsilateral and contralateral barrel field and to motor cortex areas, as well as projections to ipislateral dorsal STR and TH. Seed-based FC revealed a similar pattern, but included marked FC between both ipsilateral and contralateral hemispheres of the dorsal STR and an absence of detectable FC to the TH. For injection sites in the motor (MOs) or anterior cingulate area (ACAv), striking overlaps between SC and $\mathrm{FC}$ were found. In both instances, the regions displaying high FC extended beyond those being monosynaptically connected and included larger areas of the primary somatosensory cortex in the case of the MO seed and of the retrosplenial area in the case of the ACAv seed. Finally, an injection site/seed in the dentate gyrus revealed that $\mathrm{SC}$ was confined to the hippocampus, whereas the corresponding FC area extended across the hippocampus, cingulate, retrosplenial, ectorhinal, endorhinal, and perirhinal cortical areas, elements of the putative rodent default-mode network (DMN) (Lu et al., 2012; Sforazzini et al., 2014). This indicates that FC between some areas, in particular homologous cortical regions of the left and right hemisphere, emerges due to direct monosynaptic connections, whereas FC in spatially extended networks (e.g., DMN) indexes indirect polysynaptic connections.

To perform a comparison at a whole-brain level, we used total monosynaptic connectivity matrices reconstructed using 238 viral tracer maps (Oh et al., 2014) and matched them to seed-based FC. Injection sites were located in the isocortex $(n=98), \mathrm{HPF}$ $(n=39)$, CTXsp $(n=6)$, STR $(n=33)$, PAL $(n=8)$, and TH $(n=54)$ (for ROI list, see Fig. 3-2, available at 10.1523/ JNEUROSCI.0438-17.2017.f3-2). Ipsilateral and contralateral target regions $(n=254)$ were selected from the Allen Institute mouse brain parcellation atlas. Connectivity patterns found in the tracer injection-based SC matrix and in the seed-based FC matrices (Fig. $3 a, b$ ) confirmed the observations made using voxelwise maps; they exhibit marked similarities for cortical injection sites, showing high SC and FC with ipsilateral and contralateral isocortex, as well as with ipsilateral subcortical structures (STR, pallidum, CTXsp). However, for other specific connections, such as between isocortex and TH, FC is mostly absent despite dense monosynaptic connections. Regarding the $\mathrm{HPF}$, strong SC is observed between injection sites located in the entorhinal cortical area toward the isocortical and subcortical structures except for the TH on both the ipsilateral and contralateral side. However, this pattern was not observed in the FC matrix. Partial Spearman's rank tests confirmed the general good agreement between FC and SC for most of the selected seeds (Fig. $3 c$ ). In the isocortex, intermediate-to-strong correlations ( $\rho=$ $0.4-0.6$ ) were found with the exception of prefrontal areas (an- 


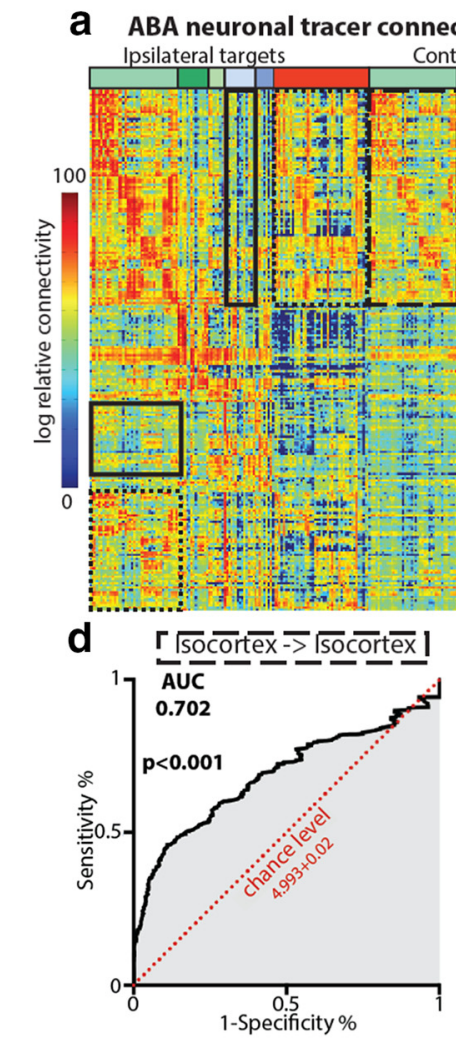

\section{mismmatx} Contralateral targets b Resting-state fMRI connectivity matrix Ipsilateral targets Contralateral targets

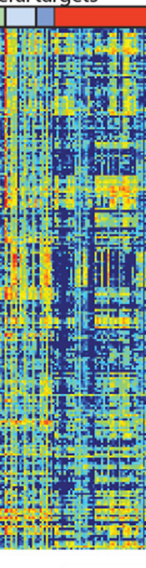

\section{e}
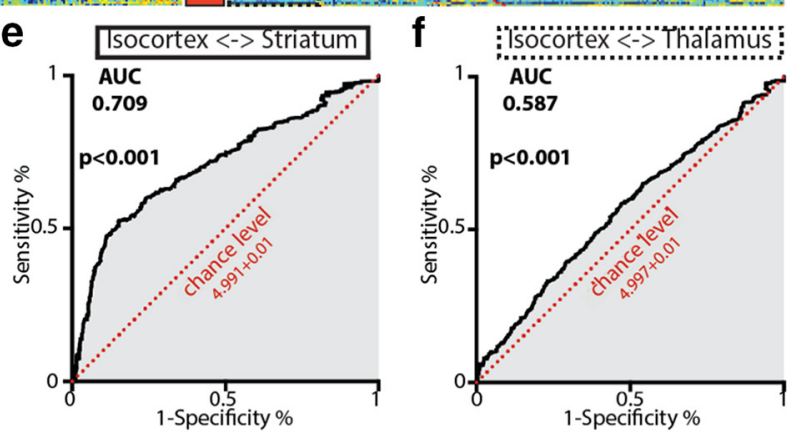

\section{Partial Spearman's rho}
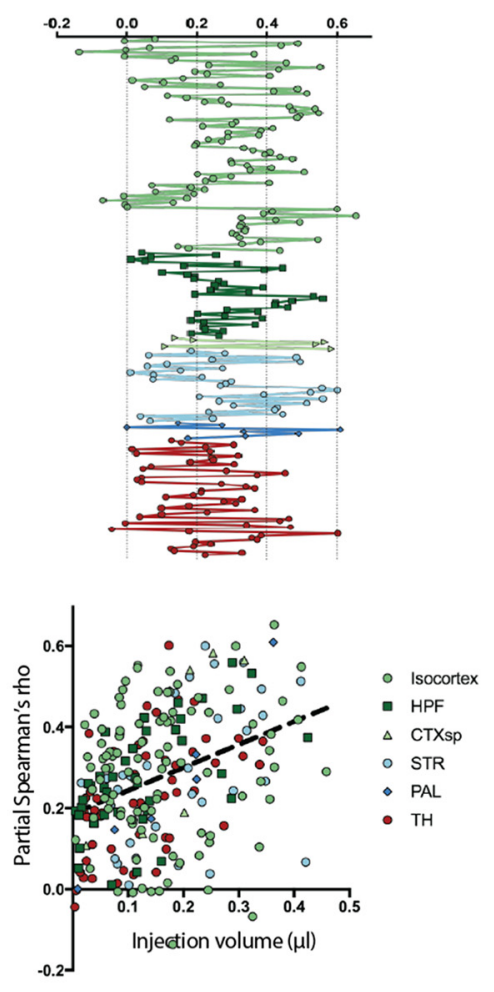

Figure 3. Comparison of the viral tracer connectivity matrix (a) and the corresponding FC matrix (b) from 238 seed-injection sites (see Fig. 3-2, available at 10.1523/JNEUROSCI.0438-17.2017. f3-2) by 254 target ROIs reveals striking similarities, in particular regarding the interactions within the isocortex. Partial Spearman's $\rho$ (corrected for target ROI volume) between structural and functional connections originating from each seed/injection experiment is displayed in c and show weak-to-intermediate (rho: $0.2-0.4$ ) and average-to-strong (rho: $0.4-0.6$ ) correlations for most of the cortical, hippocampal, and striatal seeds (colors encode for regions as shown in $\boldsymbol{a}$ and $\boldsymbol{b}$ ). Conversely, we were able to detect injection areas for which the SC-FC correlation dropped to nonsignificant levels (rho: $0-0.2$ ), notably, for injection sites in prefrontal areas (ACAd, PL, LLA, ORBI), CA1, STR, amygdala, and some thalamic nuclei, which may be driven by the relatively small volume injected and by the absence of reciprocal projections between these and other brain regions (see Fig. 3-1, available at 10.1523/JNEUROSCI.0438-17.2017.f3-1). ROC curves are shown for connectivity of isocortex $\rightarrow$ isocortex (d; dashed square boxes), isocortex $\leftrightarrow$ STR ( $\boldsymbol{e}$; black square boxes), and isocortex $\leftrightarrow$ TH ( $\boldsymbol{f}$; dotted square boxes). The AUC indicates the degree of similarity between the structural and functional metrics, ranging from 0.5 (chance level) to 1 (full similarity). Permutation testing confirmed the significant ( $>$ chance level distribution) agreement between $\mathrm{SC}$ and $\mathrm{FC}$ in all the macroscale connections, with medium to high $(>0.7)$ AUC levels for isocortex to its contralateral counterpart and for isocortex to STR and low $(<0.6)$ for isocortical to thalamic connections (see Fig. 3-3, available at 10.1523/JNEUROSCI.0438-17.2017.f3-3).

terior cingulate, prelimbic, infralimbic, and orbitofrontal areas). Conversely, most of the seed experiments of the TH showed null or weak effects $(\rho=0-0.2)$ apart from of the ventral posteromedial nuclei (see Fig. 3-2, available at 10.1523/JNEUROSCI. 0438-17.2017.f3-2). Interestingly, the degree of correlation between the SC and FC metrics were strongly significantly correlated with the amount of viral tracer injected in the structural experiments $(p<0.001)$.

The degree of similarities between SC and FC in the two metrics was further assessed within and between ontological structures by ROC analysis (Fig. $3 d-f$ and Fig. $3-3$, available at 10.1523/JNEUROSCI.0438-17.2017.f3-3). AUC estimated from the ROC analysis recapitulates the observation described above. In particular, significant correspondence exists between the isocortex injection sites/seeds and contralateral isocortex (Fig. 3d, AUC $=0.702$ ), as well as between isocortex and STR (Fig. $3 e$, AUC $=0.709$ ). For connections between TH and isocortex, correspondence between FC and SC was low, albeit significantly different from the null (i.e., chance level) distribution (Fig. $2 f$, $\mathrm{AUC}=0.587$ ) for both directionalities.

These three relevant large-scale connections were further analyzed at a voxel level for mapping the most strongly connected subareas of contralateral isocortex, STR, and TH. The HPF was not included in the detailed analysis because most of the struc- tural projections from the hippocampus were found to be confined to this brain region (Fig. 3a,b).

\section{Similar modular organization of the structural and functional} connectome in the mouse cortex

Mammalian brains are organized into distinct large-scale structures also referred as subnetworks or modules (Bullmore and Sporns, 2009; Oh et al., 2014; Zingg et al., 2014; Liska et al., 2015). The combination of structural and FC information in the same analysis space provides two independent metrics for comparing the modular organization of the brain. A winner-takes-all approach from 20 selected cortical injection seeds (Fig. $4 a$ ) revealed a remarkable correspondence between structural (Fig. $4 b$, left) and functional (Fig. $4 b$, middle) modular architecture in both the ipsilateral and contralateral isocortex. In both metrics, the somatosensory motor cortex is divided into three distinct modules, encompassing motor (Fig. $4 b$, red), sensory (Fig. $4 b$, green), and medial orbital (Fig. $4 b$, yellow) areas. A temporal associative module (Fig. $4 b$, light/dark blue) was found to encompass auditory and visual cortices and retrohippocampal regions such as the entorhinal cortex. Regions analogous to the DMN were highlighted, in particular the cingulate, prefrontal, and retrosplenial cortices (Fig. $4 b$, violet). This modular organization is consistent with subnetworks presented in previous viral tracer studies (Oh 


\section{a Winner-takes-all seeds/injection sites}

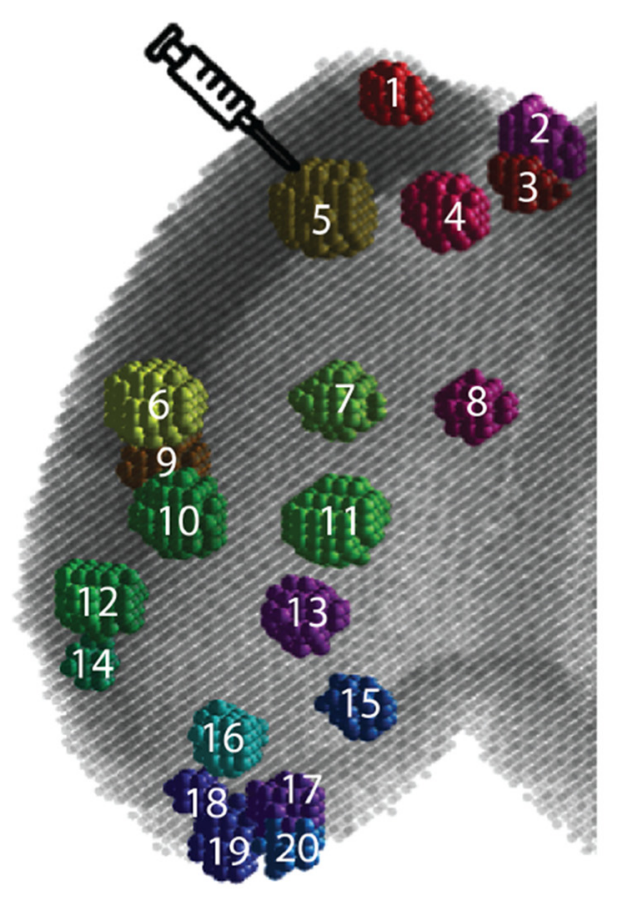

b

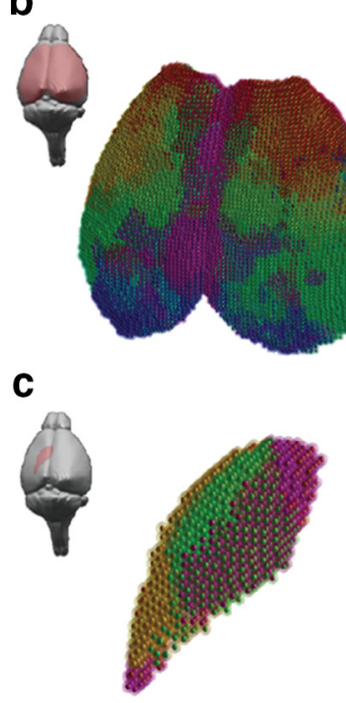

d

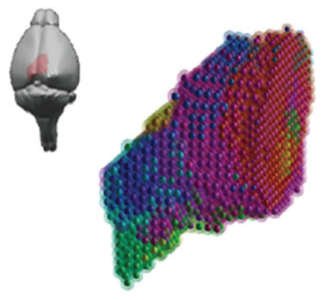

ABA tracer connectivity
Isocortex - Isocortex

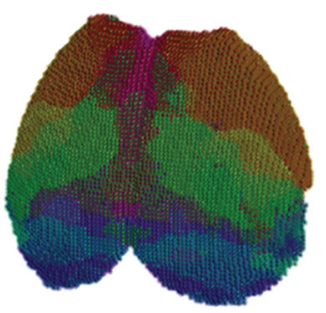

Isocortex - Striatum

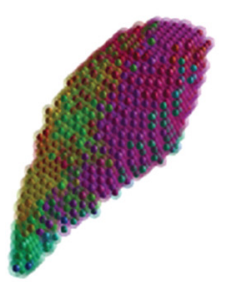

Isocortex - Thalamus

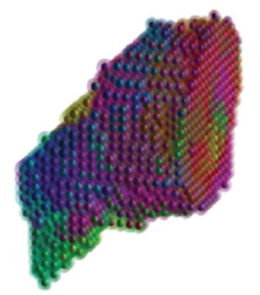

Rs-fMRI connectivity
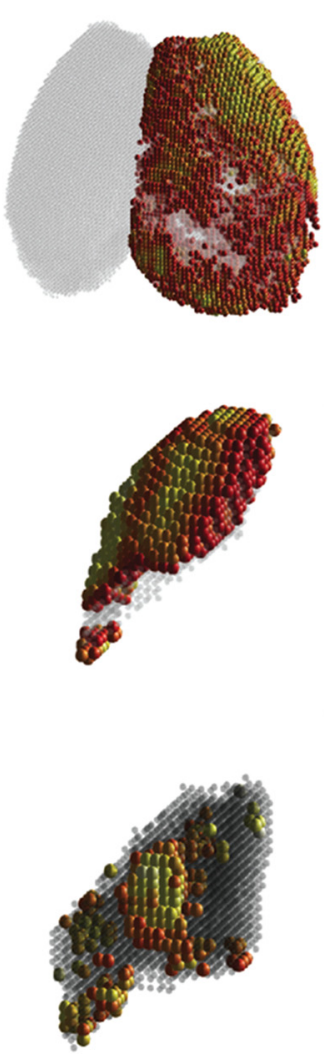

0 rarman's reorrelation Spearman's r correlation (FDR corrected)

Figure 4. Winner-takes-all analysis for 20 injection sites/seeds located in the isocortex. $\boldsymbol{a}$, Location of injection sites/seeds used for the winner-takes-all analysis mapped on a surface representation of the mouse isocortex. The labels are as follows: (1) MOs, (2) ORBm, (3) PL, (4) MOs, (5) MOp, (6) primary somatosensory area of the barrel field (SSp-bfd), (7) MOp, (8) ACAd, (9) SSs, (10) SSp-bfd, (11) SSp-II, (12) AUDd, (13) PTLp, (14) AUDd, (15) RSPagl, (16) AUDd, (17) VISp, (18) VISp, (19) VISp, and (20) VISp. Two spheres of different diameters and transparency are drawn in each voxel, indicating the first and second strongest connected injection sites/seeds originating from the isocortex toward contralateral isocortex (b), ipsilateral STR (c), and ipsilateral TH (d). Voxel-based Spearman's $r$ correlation indicates significant correlation between tracer injection and rs-fMRI data. Voxels from both isocortical and striatal maps present significant correlation (86.9\% and $87.8 \%$ of total voxels, respectively) between structural and FC. In contrast, thalamic map presents a significant correlation between the two modalities in $8.8 \%$ of the voxels only, specifically in the anteroventral and ventral posteromedial nuclei of the TH.

et al., 2014; Zingg et al., 2014). These data extend previous rsfMRI work in which the parcellation of the mouse brain produced only two cortical modules, the lateral cortical network encompassing limb and barrel field modules and the DMN, which included the temporal associative module found in this study (Liska et al., 2015).

Although the isocortex has a modular organization, it is likely that voxels (particularly voxels on the borders between modules) are not exclusively interconnected with only one module. To compare structural and FC fingerprints, we created a connectivity profile for each voxel within a mask (strength of connectivity for each injection site/seed; $n=98$ ). We then used Spearman's $\rho$ to correlate the structural and FC profile at each voxel using the data from all of the isocortical seed experiments $(n=98)$ and correcting for multiple comparisons using FDR $(p<0.05)$. This analysis highlights whether a target voxel has the same pattern of high and low connectivity for each tracer injection site and if these connectivity patterns are the same between SC and FC metrics. This analysis revealed that $86.9 \%$ of all voxels within contralateral isocortex survived FDR correction, demonstrating the high degree of overlap in connectivity fingerprints between SC and FC. Specifically, the structure-function relationship was strongest in the motor and medial orbital modules and in the DMN, whereas correlations were weaker in the area between the sensory and the temporal associative module (Fig. $4 b$, right).

\section{Matching structural and functional topographies of the cortico-striatal pathways}

Cortico-striatal projections are essential components of forebrain circuits widely involved in goal-directed behavior and motivation (Ferguson et al., 2011; Kozorovitskiy et al., 2012; Farrell et al., 2013). Hypoactivity or hyperactivity in these connections is associated with several neuropsychiatric disorders, including autism and schizophrenia (Shepherd, 2013; Ferenczi et al., 2016). From an anatomical and functional perspective, the STR is clustered into distinct domains according to input and output regions (Choi et al., 2012; Jung et al., 2014). Winner-takes-all topographical maps confirmed such a modular organization in both structural and functional datasets, which appears divided into five macro-areas densely connected with cingulate areas (Fig. $4 c$, violet), motor (Fig. $4 c$, red), sensory (Fig. $4 c$, green), medial orbital (Fig. 4c, yellow), and temporal associative (Fig. $4 c$, 


\section{a Structural connectivtiy : distance between nodes}

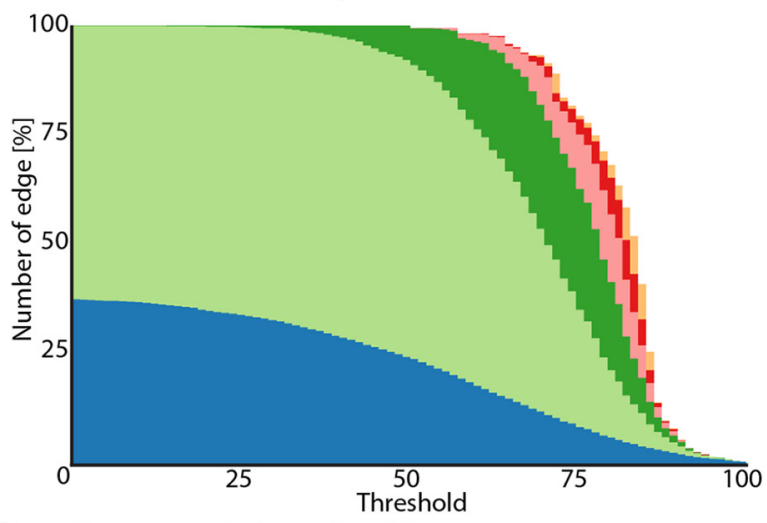

C Monosynaptic dependent FC

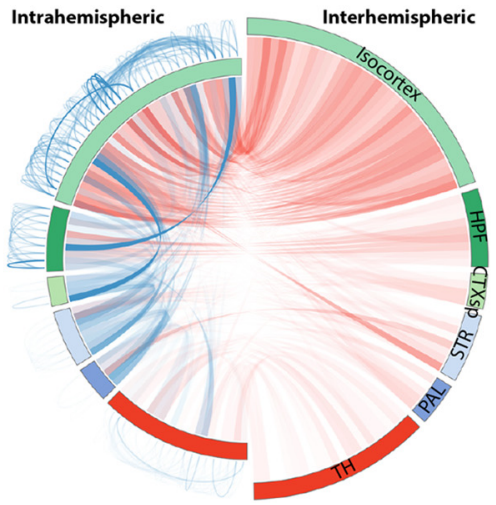

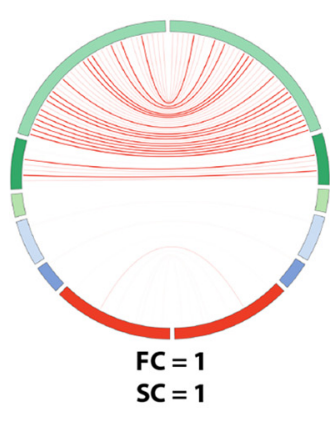

Connection likelihood $\geq 85705540 \quad 405570 \geq 85$ b Functional connectivtiy : distance between nodes

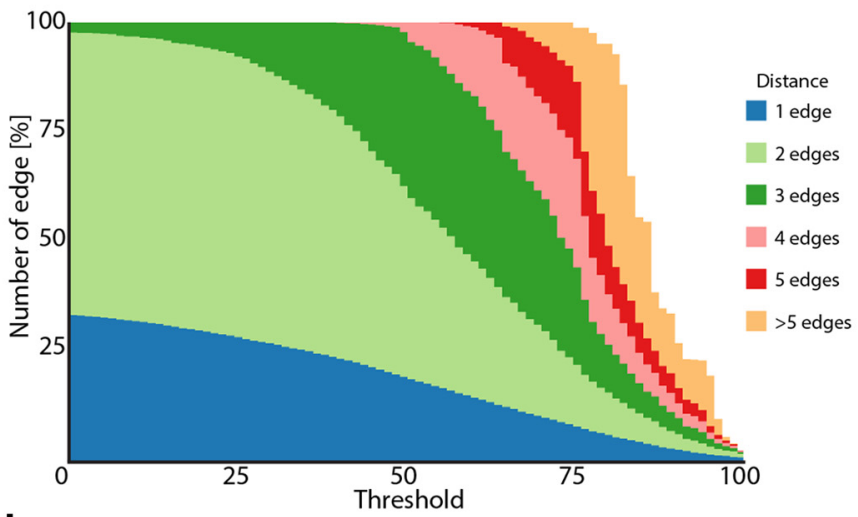

Polysynaptic dependent FC

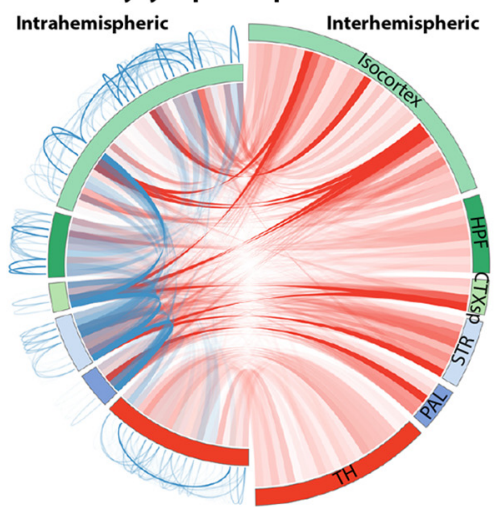

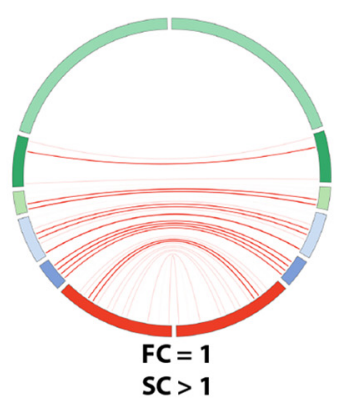

Figure 5. Distance separating node pairs from structural and FC at varying matrix threshold revealed a similar distribution $(\boldsymbol{a}, \boldsymbol{b})$. Structural and functionally connectivity matrices were normalized to a range of $0-100$. Distance was computed for both matrices with incremental threshold with step size $=1$. At a lower threshold, the number of edges separating any node pairs remains between one and two. The distance increases as threshold is increased. $\boldsymbol{c}, \boldsymbol{d}$, Distance analysis reveals monosynaptic or polysynaptic connectivity likelihood (CL) of FC. Large circular plots show transparencycoded links that represent $\mathrm{CL}$ for intrahemispheric (blue) and interhemispheric (red) connections across the brain. For the sake of clarity, intrahemispheric connections within brain structures are plotted outside and inside of the circle labeling the major brain regions (left side of graphs). Smaller circular plots indicate links between bilateral homotopic region pairs only. c, Isocortex presents balanced intrahemispheric and interhemispheric monosynaptic CL, toward HPF, (TXsp, and STR (intrahemispheric) and toward contralateral homotopic ROI (interhemispheric). $\boldsymbol{d}$, Polysynaptic CL presents more diverse links between ROIs from different ontological structures and hemispheres, for example, STR to contralateral isocortex and STR. Polysynaptic homotopic interactions are found in the CTXsp, STR, PAL, and TH. Likelihood values are given as percentages. Mismatches between structural FC are shown in Figure 5-1 (available at 10.1523/JNEUROSCI.0438-17.2017.f5-1).

blue) cortices. The partition of the ipsilateral STR presented remarkable similarities with modular divisions found with viral tracers (Oh et al., 2014; Hintiryan et al., 2016). Remarkably, $87.8 \%$ of striatal voxels showed a significant Spearman's correlation between anatomical and functional architecture, with the highest degree of correlation found in the motor cortex to dorsal STR pathway.

\section{Lack of FC for networks involving the TH}

The TH is a highly heterogeneous structure subdivided into distinct nuclei that are connected to the cerebral cortex through multiple anatomical loops and that process sensory, limbic, and heteromodal information (Behrens et al., 2003). Detailed maps of human and rodent cortico-thalamic cytoarchitecture are available (Jones, 2007; Oh et al., 2014). In humans, imaging data suggest an overall correspondence between structural diffusion data and functional imaging, but this has been only demonstrated at the macroscale level (Zhang et al., 2010). Our results revealed minimal correspondence of FC and SC between isocortex and ipsilateral TH. Statistically, only $8.8 \%$ of all voxels within the ipsilateral TH showed a significant overlap of connectivity profiles (Fig. 4d). Specifically, these areas of good correspondence reflect the connections between the anteroventral nuclei of the
TH with retrosplenial cortex (Fig. $4 d$, purple) and between the ventral posteromedial nuclei with somatosensory-barrel field cortex (Fig. $4 d$, green). For all other nuclei, the overall structural monosynaptic connections derived from viral tracing were not reflected by the functional data as measured with rs-fMRI.

\section{Monosynaptic and polysynaptic dependency of resting-state networks}

Our comparison of the SC and FC matrices revealed both areas of similarities and divergences between the two connectivity measures. In a final set of analyses, we determined the minimum number of edges, or connections, separating two nodes (ROIs). This analysis was run for both structural and FC matrices. The number of edges necessary to connect two nodes was used to divide structural-FC relationships into three categories: (1) monosynaptic FC; (2) polysynaptic FC; and (3) low FC despite monosynaptic SC, which were labeled "mismatched."

The two distance metrics showed a similar distribution. For low threshold values, most of the ROIs were found to be separated by one or two edges (Fig. $5 a, b$ ). As expected, the distance between node pairs increased with higher threshold values, consistent with increasing sparsity of the matrices. High monosynaptic FC likelihood values were obtained between the isocortex 
and the ipsilateral HPF and STR (Fig. 5c). Notably, high interhemispheric monosynaptic connectivity likelihood was also found between bilateral homotopic isocortices and, to a lesser extent, between HPFs, as indicated by the plot restricted to contralateral homotopic ROIs (Fig. 5c, left). Polysynaptic connectivity likelihood was found to be more diverse in terms of interactions between the ontological structures and hemispheres (Fig. $5 d$ ). For instance, there were notable interhemispheric projections from the STR toward the isocortex and the contralateral STR, as well as between homotopic subcortical regions in both hemispheres (Fig. $5 d$, left). In summary, we found that functional networks in the isocortex were predominantly monosynaptic. Conversely, subcortical networks present more diverse projections and rely in several instances on polysynaptic projections. Mismatched FC is mostly present in edges between thalamic and ROIs in the cortex, HPF, and STR, consistent with the notions of weak thalamic FC observed above (see Fig. 5-1, available at 10.1523/JNEUROSCI.0438-17.2017.f5-1).

\section{Discussion}

Here, we report the brain-wide functional connectome of the mouse and analyze how circuits of enhanced long-range FC map onto monosynaptic structural pathways. We confirmed that FC emerges preferably along monosynaptic connections, for example, between homotopic isocortical areas across hemispheres. We further showed that rs-fMRI is an excellent tool for studying cortico-striatal subcircuits, which match monosynaptic anatomical connectivity. Last, we identified specific networks (e.g., high FC between left and right STR and TH) where rs-fMRI oscillations synchronize via polysynaptic pathways.

Understanding the organizational principles underlying the structure-function relationship of the brain has been a central question in neuroscience. Converging evidence across species has confirmed fMRI measurements reflect neuronal activity both for stimulus-evoked and rs-fMRI (Logothetis et al., 2001; Mantini et al., 2007; Schölvinck et al., 2010). Moreover, the topology of human FC networks corresponds well to major structural tracts forming cortico-cortical (Hagmann et al., 2008; Honey et al., 2009; van den Heuvel et al., 2009), cortico-striatal (Jarbo and Verstynen, 2015) or cortico-thalamic (Zhang et al., 2010) circuits. However, many of these studies have been using information derived from diffusion-weighted imaging to reconstruct SC networks. Unfortunately, there are still a number of limitations with diffusion imaging, including the limited spatial resolution, low sensitivity in gray matter, and difficulties with identifying fiber crossings and terminations. Tracer information in the mouse, by contrast, allows one to reconstruct SC networks with greater spatial resolution and without ambiguity with respect to fiber crossing or monosynaptic and polysynaptic projections. Previous work comparing viral tracer to functional connectivity have, like our work, reported systematically good correspondence between SC and FC across the whole brain (Stafford et al., 2014), though these studies do not systematically investigate SC-FC relationships among various networks within the connectome or have focused only on specific networks such as the hippocampus (Bergman et al., 2016). We have been able to demonstrate structure-function correspondence using the proven hardwired connections between the nodes involved in a wide range of resting-state networks, many of which correspond to human networks (Sforazzini et al., 2014).

Resting-state FC networks in humans and other species have shown a preponderant bilateral organization (Damoiseaux et al., 2006), with the exception of right and left frontoparietal net- works in the human brain (van den Heuvel et al., 2009). Previous studies investigating resting-state networks in the rodent brain have also demonstrated this bilateral organization (Grandjean et al., 2014; Sforazzini et al., 2014; Zerbi et al., 2015), a property lost or greatly reduced in mice presenting with agenesis of the corpus callosum, supporting the notion that axonal projections are causally involved in supporting distal FC (Sforazzini et al., 2016; Schroeter et al., 2017). Many of these networks have been proposed to be analogous to human resting-state networks, such as the DMN and the salience network (Upadhyay et al., 2011; Lu et al., 2012; Sforazzini et al., 2014). An interesting feature of rodent FC is the presence of robust striatal functional networks, which we have repeatedly found to be divided into three entities, the dorsal and lateral STR mostly overlapping with the caudate and putamen and ventral STR overlapping with nucleus accumbens (Grandjean et al., 2014; Zerbi et al., 2015). This segregation into specific circuits is interesting because they are affected differentially in murine models of brain disorders related to substance abuse (Hyman et al., 2006), movement disorders (Poston and Eidelberg, 2012), major depression (Kerestes et al., 2015), Parkinson's disease (Rolinski et al., 2015), and schizophrenia (Sorg et al., 2013; for review, see Shepherd, 2013). One specific property of these networks is that, compared with cortical networks, which rely strongly on direct projections, their bilateral organization relies on polysynaptic relays, either via the cortico-striatal or nigrostriatal/mesolimbic pathways (Ferenczi et al., 2016). Therefore, the preponderant position of striatal networks among the rodent resting-state functional networks and the availability of mouse models of brain disorders and substance abuse offer new opportunities to study the large-scale functional implications of disease on these specific networks.

In addition to highlighting the significant overlap between structural and FC, we also identified some networks where structural and FC did not match, specifically the cortico-thalamic projections. This might be attributed to effects of anesthesia. Although controlled anesthesia and mechanical ventilation are expected to increase the robustness and reproducibility of the functional readout by limiting drastically physiological noise through reduced motion and constant breathing cycle, anesthesia agents will also affect neuronal networks. Medetomidine for example, albeit kept to a minimal level, has been reported to interfere with cortico-thalamic FC (Fukuda et al., 2013), particularly in connection to rs-fMRI studies (Grandjean et al., 2014; Nasrallah et al., 2014), specifically affecting regions expressing high levels of $\alpha$-2 adrenergic receptors, the target of medetomidine, in a dose-dependent fashion (Nasrallah et al., 2014). Another possible confounding factor is the relatively small injection volume used to target the thalamic nuclei, an effect further exacerbated by the relative small ROI size of these corresponding nuclei. In fact, 22 of 54 thalamic areas $(41 \%)$ were injected with $<0.1 \mu$ l of viral tracer. In comparison, only $28 \%$ of isocortical and $24 \%$ of striatal injections received $<0.1 \mu \mathrm{l}$ of tracer. The higher anatomically specificity obtained with small injections is desired for small nuclei such as in the TH. However, this is usually achieved at the cost of a reduced transcriptional efficiency of the virus and therefore a lower sensitivity of the measure; this may have introduced a systematic noise in the connectivity derived to macroscale ontological targets. Moreover, this effect is exacerbated by the relatively low resolution of fMRI, $\sim 300 \mu \mathrm{m}^{3}$, which does not permit the resolution of smaller thalamic nucleus separately. Notably, our results showed a robust correlation between the SC-FC Spearman's $\rho$ and the injection volume, which corroborates these findings. This suggests that thalamic resting-state networks in 
anesthetized rodents should be interpreted with caution because their interpretation in terms of SC may be confounded by intrinsic shortcomings in the measurements.

The comparison between the two metrics remains limited in some aspects. First, in mouse studies, anesthesia was shown to interfere with brain function and therefore to affect FC readouts as discussed for cortico-thalamic connectivity. Recent work has compared awake rs-fMRI with viral tracer; however, distal FC estimated in this latter work was relatively low with respect to the selected seeds (Bergmann et al., 2016) compared with results reported with the optimized anesthesia protocol used in this study (Grandjean et al., 2014). This corroborates previous reports indicating difficulties in adapting rat awake protocols to mice (Jonckers et al., 2014). In addition to displaying strong and robust distal FC, the anesthesia protocol was shown to recapitulate advantages of both isoflurane and medetomidine protocols, that is, strong cortical and subcortical FC, respectively, while displaying minimal undesirable effects.

Second, a study in monkeys has shown that mild and deep anesthesia was associated with greater correspondence between FC and SC, whereas the awake state captured rich FC patterns beyond that predicted with anatomical projections, including patterns of dynamic FC (Barttfeld et al., 2015). Although it remains difficult to compare anesthesia depth across studies and more so across species, the present study uses a light anesthesia/ sedation protocol, which retains anti-correlation (Grandjean et al., 2014) and rich patterns of dynamic FC (Grandjean et al., 2017) comparable to results described in the awake monkey. This may explain the presence of rich polysynaptic-dependent FC observed in the present study.

Third, tracer-based SC from the Allen Institute contains directional information between any two ROIs, whereas FC captured only the shared information between the time series regardless of the directionality. This may have biased the estimation of the correspondence between the two metrics, although recent studies showed a general good correspondence between anterograde and retrograde pathways in the mouse (Zingg et al., 2014). Indeed, most anatomical projections are coupled with reciprocal projections that might offset directionality effect in the SC matrices. The hippocampus is a notable exception to this rule because most projections are unidirectional and inputs to the hippocampus come exclusively from the para-HPF; therefore, distal FC with respect to the hippocampus, as with the other elements of the rodent DMN, is mostly exclusively of polysynaptic nature. Applying causal models such as Granger causality or dynamic causal modeling (Li et al., 2011) to resting-state networks may provide information regarding directionality and thus provide further information regarding the correspondence between structure and function.

Whole-brain comparison of SC estimated from tracer-based reconstruction and FC from rs-fMRI obtained in the mouse revealed substantial agreement between the two metrics across several levels from individual connectivity maps derived from injection sites/seeds, to whole-brain interactions, and to modular organization of SC-FC. This close correspondence between FC and SC forms the basis for linking rs-fMRI in the mouse to its anatomical underpinnings, providing a strong foundation to investigate the structure/function relationship and its alteration due to disease within networks. Our findings also form the basis for rodent fMRI in combination with pharmacogenetically/optogenetically controlled manipulation to dissect the role of selected cellular populations in subnetworks (Lee et al., 2010), thus allowing the resolution of a cell-specific mechanism taking place in large-scale networks in the healthy and diseased brain. These approaches may shine new light onto the organization of the healthy brain and onto specific neuronal alterations underlying brain disorders.

\section{References}

Barttfeld P, Uhrig L, Sitt JD, Sigman M, Jarraya B, Dehaene S (2015) Signature of consciousness in the dynamics of resting-state brain activity. Proc Natl Acad Sci U S A 112:887-892. CrossRef Medline

Basser PJ, Mattiello J, LeBihan D (1994) MR diffusion tensor spectroscopy and imaging. Biophys J 66:259-267. CrossRef Medline

Behrens TE, Johansen-Berg H, Woolrich MW, Smith SM, Wheeler-Kingshott CA, Boulby PA, Barker GJ, Sillery EL, Sheehan K, Ciccarelli O, Thompson AJ, Brady JM, Matthews PM (2003) Non-invasive mapping of connections between human thalamus and cortex using diffusion imaging. Nat Neurosci 6:750-757. CrossRef Medline

Bergmann E, Zur G, Bershadsky G, Kahn I (2016) The organization of mouse and human cortico-hippocampal networks estimated by intrinsic functional connectivity. Cereb Cortex 26:4497-4512. CrossRef Medline

Biswal BB et al. (2010) Toward discovery science of human brain function. Proc Natl Acad Sci U S A 107:4734-4739. CrossRef Medline

Biswal B, Yetkin FZ, Haughton VM, Hyde JS (1995) Functional connectivity in the motor cortex of resting human brain using echo-planar MRI. Magn Reson Med 34:537-541. CrossRef Medline

Bullmore E, Sporns O (2009) Complex brain networks: graph theoretical analysis of structural and functional systems. Nat Rev Neurosci 10:186198. CrossRef Medline

Calabrese E, Badea A, Cofer G, Qi Y, Johnson GA (2015) A diffusion MRI tractography connectome of the mouse brain and comparison with neuronal tracer data. Cereb Cortex 25:4628-4637. CrossRef Medline

Choi EY, Yeo BT, Buckner RL (2012) The organization of the human striatum estimated by intrinsic functional connectivity. J Neurophysiol 108: 2242-2263. CrossRef Medline

Cohen J (1988) Statistical power analysis for the behavioral sciences. Hillsdale, NJ: Erlbaum.

Damoiseaux JS, Rombouts SA, Barkhof F, Scheltens P, Stam CJ, Smith SM, Beckmann CF (2006) Consistent resting-state networks across healthy subjects. Proc Natl Acad Sci U S A 103:13848-13853. CrossRef Medline

De Luca M, Beckmann CF, De Stefano N, Matthews PM, Smith SM (2006) fMRI resting state networks define distinct modes of long-distance interactions in the human brain. Neuroimage 29:1359-1367. CrossRef Medline

Farrell MS, Pei Y, Wan Y, Yadav PN, Daigle TL, Urban DJ, Lee HM, Sciaky N, Simmons A, Nonneman RJ, Huang XP, Hufeisen SJ, Guettier JM, Moy SS, Wess J, Caron MG, Calakos N, Roth BL (2013) A Galphas DREADD mouse for selective modulation of cAMP production in striatopallidal neurons. Neuropsychopharmacology 38:854-862. CrossRef Medline

Ferenczi EA, Zalocusky KA, Liston C, Grosenick L, Warden MR, Amatya D, Katovich K, Mehta H, Patenaude B, Ramakrishnan C, Kalanithi P, Etkin A, Knutson B, Glover GH, Deisseroth K (2016) Prefrontal cortical regulation of brainwide circuit dynamics and reward-related behavior. Science 351:aac9698. CrossRef Medline

Ferguson SM, Eskenazi D, Ishikawa M, Wanat MJ, Phillips PE, Dong Y, Roth BL, Neumaier JF (2011) Transient neuronal inhibition reveals opposing roles of indirect and direct pathways in sensitization. Nat Neurosci 14:2224. CrossRef Medline

Fukuda M, Vazquez AL, Zong X, Kim SG (2013) Effects of the alpha(2)adrenergic receptor agonist dexmedetomidine on neural, vascular and BOLD fMRI responses in the somatosensory cortex. Eur J Neurosci 37: 80-95. CrossRef Medline

Grandjean J, Schroeter A, Batata I, Rudin M (2014) Optimization of anesthesia protocol for resting-state fMRI in mice based on differential effects of anesthetics on functional connectivity patterns. Neuroimage 102:838 847. CrossRef Medline

Grandjean J, Preti MG, Bolton TAW, Buerge M, Seifritz E, Pryce CR, Van De Ville D, Rudin M (2017) Dynamic reorganization of intrinsic functional networks in the mouse brain. Neuroimage 152:497-508. CrossRef Medline

Hagmann P, Cammoun L, Gigandet X, Meuli R, Honey CJ, Wedeen VJ, Sporns O (2008) Mapping the structural core of human cerebral cortex. PLoS Biol 6:e159. CrossRef Medline

Hintiryan H, Foster NN, Bowman I, Bay M, Song MY, Gou L, Yamashita S, Bienkowski MS, Zingg B, Zhu M, Yang XW, Shih JC, Toga AW, Dong HW 
(2016) The mouse cortico-striatal projectome. Nat Neurosci 19:11001114. CrossRef Medline

Honey CJ, Sporns O, Cammoun L, Gigandet X, Thiran JP, Meuli R, Hagmann P (2009) Predicting human resting-state functional connectivity from structural connectivity. Proc Natl Acad Sci U S A 106:2035-2040. CrossRef Medline

Hyman SE, Malenka RC, Nestler EJ (2006) Neural mechanisms of addiction: the role of reward-related learning and memory. Annu Rev Neurosci 29:565-598. CrossRef Medline

Jarbo K, Verstynen TD (2015) Converging structural and functional connectivity of orbitofrontal, dorsolateral prefrontal, and posterior parietal cortex in the human striatum. J Neurosci 35:3865-3878. CrossRef Medline

Jbabdi S, Sotiropoulos SN, Haber SN, Van Essen DC, Behrens TE (2015) Measuring macroscopic brain connections in vivo. Nat Neurosci 18: 1546-1555. CrossRef Medline

Jonckers E, Delgado y Palacios R, Shah D, Guglielmetti C, Verhoye M, Van der Linden A (2014) Different anesthesia regimes modulate the functional connectivity outcome in mice. Magn Reson Med 72:1103-1112. CrossRef Medline

Jones EG (2007) The thalamus, Ed 2. Cambridge: Cambridge University.

Jung WH, Jang JH, Park JW, Kim E, Goo EH, Im OS, Kwon JS (2014) Unravelling the intrinsic functional organization of the human striatum: a parcellation and connectivity study based on resting-state FMRI. PLoS One 9:e106768. CrossRef Medline

Kasthuri N, Lichtman JW (2007) The rise of the 'projectome'. Nat Methods 4:307-308. CrossRef Medline

Kerestes R, Harrison BJ, Dandash O, Stephanou K, Whittle S, Pujol J, Davey CG (2015) Specific functional connectivity alterations of the dorsal striatum in young people with depression. Neuroimage Clin 7:266-272. CrossRef Medline

Kozorovitskiy Y, Saunders A, Johnson CA, Lowell BB, Sabatini BL (2012) Recurrent network activity drives striatal synaptogenesis. Nature 485: 646-650. CrossRef Medline

Kundu P, Inati SJ, Evans JW, Luh WM, Bandettini PA (2012) Differentiating BOLD and non-BOLD signals in fMRI time series using multi-echo EPI. Neuroimage 60:1759-1770. CrossRef Medline

Lee JH, Durand R, Gradinaru V, Zhang F, Goshen I, Kim DS, Fenno LE, Ramakrishnan C, Deisseroth K (2010) Global and local fMRI signals driven by neurons defined optogenetically by type and wiring. Nature 465:788-792. CrossRef Medline

Li B, Daunizeau J, Stephan KE, Penny W, Hu D, Friston K (2011) Generalised filtering and stochastic DCM for fMRI. Neuroimage 58:442-457. CrossRef Medline

Lichtman JW, Livet J, Sanes JR (2008) A technicolour approach to the connectome. Nat Rev Neurosci 9:417-422. CrossRef Medline

Liska A, Galbusera A, Schwarz AJ, Gozzi A (2015) Functional connectivity hubs of the mouse brain. Neuroimage 115:281-291. CrossRef Medline

Logothetis NK, Pauls J, Augath M, Trinath T, Oeltermann A (2001) Neurophysiological investigation of the basis of the fMRI signal. Nature 412: 150-157. CrossRef Medline

Lu H, Zou Q, Gu H, Raichle ME, Stein EA, Yang Y (2012) Rat brains also have a default mode network. Proc Natl Acad Sci U S A 109:3979-3984. CrossRef Medline

Mantini D, Perrucci MG, Del Gratta C, Romani GL, Corbetta M (2007) Electrophysiological signatures of resting state networks in the human brain. Proc Natl Acad Sci U S A 104:13170-13175. CrossRef Medline

Nasrallah FA, Tay HC, Chuang KH (2014) Detection of functional connectivity in the resting mouse brain. Neuroimage 86:417-424. CrossRef Medline

Oh SW et al. (2014) A mesoscale connectome of the mouse brain. Nature 508:207-214. CrossRef Medline
Park HJ, Friston K (2013) Structural and functional brain networks: from connections to cognition. Science 342:1238411. CrossRef Medline

Poston KL, Eidelberg D (2012) Functional brain networks and abnormal connectivity in the movement disorders. Neuroimage 62:2261-2270. CrossRef Medline

Rolinski M, Griffanti L, Szewczyk-Krolikowski K, Menke RA, Wilcock GK, Filippini N, Zamboni G, Hu MT, Mackay CE (2015) Aberrant functional connectivity within the basal ganglia of patients with Parkinson's disease. Neuroimage Clin 8:126-132. CrossRef Medline

Schölvinck ML, Maier A, Ye FQ, Duyn JH, Leopold DA (2010) Neural basis of global resting-state fMRI activity. Proc Natl Acad Sci U S A 107:10238 10243. CrossRef Medline

Schroeter A, Grandjean J, Schlegel F, Saab BJ, Rudin M (2017) Contributions of structural connectivity and cerebrovascular parameters to functional magnetic resonance imaging signals in mice at rest and during sensory paw stimulation. J Cereb Blood Flow Metab 37:2368-2382. CrossRef Medline

Sethi SS, Zerbi V, Wenderoth N, Fornito A, Fulcher BD (2017) Structural connectome topology relates to regional BOLD signal dynamics in the mouse brain. Chaos 27:047405. CrossRef Medline

Sforazzini F, Schwarz AJ, Galbusera A, Bifone A, Gozzi A (2014) Distributed BOLD and CBV-weighted resting-state networks in the mouse brain. Neuroimage 87:403-415. CrossRef Medline

Sforazzini F, Bertero A, Dodero L, David G, Galbusera A, Scattoni ML, Pasqualetti M, Gozzi A (2016) Altered functional connectivity networks in acallosal and socially impaired BTBR mice. Brain Struct Funct 221:941954. CrossRef Medline

Shepherd GM (2013) Corticostriatal connectivity and its role in disease. Nat Rev Neurosci 14:278-291. CrossRef Medline

Sorg C, Manoliu A, Neufang S, Myers N, Peters H, Schwerthöffer D, Scherr M, Mühlau M, Zimmer C, Drzezga A, Förstl H, Bäuml J, Eichele T, Wohlschläger AM, Riedl V (2013) Increased intrinsic brain activity in the striatum reflects symptom dimensions in schizophrenia. Schizophr Bull 39:387395. CrossRef Medline

Stafford JM, Jarrett BR, Miranda-Dominguez O, Mills BD, Cain N, Mihalas S, Lahvis GP, Lattal KM, Mitchell SH, David SV, Fryer JD, Nigg JT, Fair DA (2014) Large-scale topology and the default mode network in the mouse connectome. Proc Natl Acad Sci U S A 111:18745-18750. CrossRef Medline

Swanson LW (1982) The projections of the ventral tegmental area and adjacent regions: a combined fluorescent retrograde tracer and immunofluorescence study in the rat. Brain Res Bull 9:321-353. CrossRef Medline

Upadhyay J, Baker SJ, Chandran P, Miller L, Lee Y, Marek GJ, Sakoglu U, Chin CL, Luo F, Fox GB, Day M (2011) Default-mode-like network activation in awake rodents. PLoS One 6:e27839. CrossRef Medline

van den Heuvel MP, Mandl RC, Kahn RS, Hulshoff Pol HE (2009) Functionally linked resting-state networks reflect the underlying structural connectivity architecture of the human brain. Hum Brain Mapp 30:31273141. CrossRef Medline

van den Heuvel MP, Bullmore ET, Sporns O (2016) Comparative connectomics. Trends Cogn Sci 20:345-361. CrossRef Medline

Zerbi V, Grandjean J, Rudin M, Wenderoth N (2015) Mapping the mouse brain with rs-fMRI: An optimized pipeline for functional network identification. Neuroimage 123:11-21. CrossRef Medline

Zhang D, Snyder AZ, Shimony JS, Fox MD, Raichle ME (2010) Noninvasive functional and structural connectivity mapping of the human thalamocortical system. Cereb Cortex 20:1187-1194. CrossRef Medline

Zingg B, Hintiryan H, Gou L, Song MY, Bay M, Bienkowski MS, Foster NN, Yamashita S, Bowman I, Toga AW, Dong HW (2014) Neural networks of the mouse neocortex. Cell 156:1096-1111. CrossRef Medline 